http://jmscr.igmpublication.org/home/ ISSN (e)-2347-176x ISSN (p) 2455-0450 crossref DOI: https://dx.doi.org/10.18535/jmscr/v8i2.47

\author{
Dournal Of Medical Science And Clinical Research \\ IGM Publication \\ An official Publication of IGM Publication
}

\title{
Risk Factor for Incident Renal Stone: A Tertiary Centre Experience
}

\author{
Authors \\ Rajesh Waikhom $^{1^{*}}$, Boboy Thounaojam ${ }^{2}$ \\ Department of Nephrology, Jawaharlal Nehru institute of Medical Sciences -Porompat \\ *Corresponding Author \\ Dr Rajesh Waikhom
}

Associate Professor, Department of Nephrology (Jawaharlal Nehru Institute of Medical Sciences)

Abstract
Renal stone continues to be a leading cause of chronic kidney disease. Renal stone recurs at high variable
rate. There are certain predisposing factors identified for formation of kidney stones. In this retrospective
study we identify the risk factor for incident renal stone formation in our local population. Hypocitraturia
was the most common predisposing factor and was seen in 95 percent of the patient. The hypocitraturia did
not normalize with the standard dosage of citrate supplementation in majority of the patient.

\section{Introduction}

Renal stone and its complication is a significant cause of morbidity and mortality in the general population. Once a renal stone is formed they tend to recur in a sizable proportion of patient. It is important to identify the risk factor for stone formation so that the risk of recurrence is reduced. In this study we attempt to find the predisposing risk factor for renal stone in our local population.

\section{Material and Methods}

This is a retrospective study with data compiled from a large tertiary government hospital in Imphal.

Inclusion Criteria: Adult patients with newly diagnosed renal stone were included in this study. Patients were recruited from Nephrology department of Jawaharlal Nehru Institute of Medical sciences which serve as a referral centre. The study period was from February 2014 till February 2019 .The patients were subjected to the following test as a work up for renal stone:
Urine examination to look for $\mathrm{pH}$. Urine microscopic examination. 24 hour urine estimation of urine citrate /uric acid/calcium/ oxalate .Kidney function test, Complete haemogram, intact parathyroid hormone ,serum bicarbonate were done in all the patients .Nuclear scan of parathyroid gland was performed in suspected case of primary hyperparathyroidism .Stone analysis was done whenever the stone was retrieved.

Hypercalciuria is defined as a 24 hour urine calcium values exceeding more than $300 \mathrm{mg}$ in males and $250 \mathrm{mg}$ in females.

Hyperoxaluria is defined as a $24 \mathrm{hr}$ urine oxalate values exceeding more than $40 \mathrm{mg}$.

Hyperuricosuria is defined as a 24 hour urine uric acid more than $800 \mathrm{mg}$ in male and $750 \mathrm{mg}$ in females.

Hypocitraturia is defined as a 24 hour urine citrate amount less than $320 \mathrm{mg}$.

Exclusion Criteria: Patients with deranged renal function (serum creatinine more than $1.5 \mathrm{mg} / \mathrm{dl}$ ) 
were excluded. Patients with polycystic kidney disease or those with significant congenital urinary tract anomalies and inborn errors of metabolism were excluded.

\section{Statistical Analysis}

Continuous variables are expressed as means. SPSS 16 statistical software was used in the data analysis.

\section{Results}

102 patients with newly diagnosed renal stone disease were included during the study period .24 hour urine biochemistry results were available in 80 patients. Stone analysis results were available in 12 patients. The findings are as shown in table 2.

Table 1 Baseline characteristics of the patient

\begin{tabular}{|l|c|}
\hline & \\
\hline Age & $45.4+/-16.33$ \\
\hline Sex(M/F) & $74 / 28$ \\
\hline Dietary pattern & 88 \\
$\begin{array}{l}\text { Non vegetarian diet } \\
\text { Vegetarian diet calcium }\end{array}$ & $9.13+/-0.13$ \\
\hline $\begin{array}{l}\text { Serum } \\
\text { (corrected for albumin) }\end{array}$ & $1.12+/-0.17$ \\
\hline Serum creatinine & \\
\hline
\end{tabular}

Table 2: Baseline characteristic of the risk factor

\begin{tabular}{|l|c|c|}
\hline & Number of patients & Mean value \\
\hline Intact parathyroid hormone level & & $22.2+/-28.6$ \\
Number of patients who had parathyroid level greater than & & \\
upper limit & 4 & \\
Confirmed hyperparathyroidism on nuclear scan & 1 & \\
\hline Distal renal tubular acidosis & None & \\
\hline Hyperoxaluria (no. of patients) & $10 / 80$ & $27.5+/-15.3$ \\
\hline Hypercalciuria & $5 / 80$ & $135.05+/-80.4$ \\
\hline Hypocitraturia & $76 / 80$ & $47.6+/-32.11$ \\
\hline Hyperuricosuria & $2 / 80$ & $381.4+/-141.8$ \\
\hline Stone analysis :12 patients & $\begin{array}{c}\text { Calcium oxalate } 10 \\
\text { Mixed stone } 1\end{array}$ & \\
& Uric acid 1 & \\
\hline
\end{tabular}

\section{Discussion}

Renal stone and its related complication is the second most common cause of chronic kidney disease in our local population. Renal stone can cause obstructive nephropathy with renal failure. It contributes to urosepsis and can also cause chronic tubulointerstitial nephritis .Renal stone have also been associated with cardiovascular disease and bone disease ${ }^{(1,2)}$.

Renal stones are known to recur at a high rate ${ }^{(3)}$. The cause of recurrence is because the primary predisposing factor remained uncorrected .As such it is important to identify the risk factor in order to reduce the risk of recurrence. Once the risk factors are identified then proper corrective measures can be adopted.

In our study population, the most common risk factor for stone formation was hypocitraturia which was noted in 95 percent of $(76 / 80)$ the patient. The mean urinary citrate level was 47.6 +/- 32.11. Urinary citrate complexes with calcium and prevent calcium super saturation as well as nucleation of calcium oxalate and calcium phosphate complexes. Approximately $65-90 \%$ of filtered load of citrate is reabsorbed ${ }^{(4,5)}$. Citrate are absorbed through the apical membrane from proximal renal tubules which is believed to be $\mathrm{pH}$ dependent. The tubular citrate exists mostly as citrate -3 ion although it is reabsorbed as citrate -2 ion through the sodium citrate co transporter. With decreasing tubular $\mathrm{pH}$, citrate reabsorption increases ${ }^{(6)}$.

The most common cause for hypocitraturia is idiopathic. Other factors include distal renal tubular acidosis ${ }^{(7,8)}$, chronic diarrhoea, gastrointestinal disorders ${ }^{(9,10)}$ and high animal protein intake $e^{(11,12)}$. Certain medications are also known to affect urinary citrate excretion ${ }^{(13,14,15)}$. In our population we could not find any case of obvious distal renal tubular acidosis. Patients were not subjected to evaluation for incomplete distal renal tubular acidosis .In the absence of any other 
obvious predisposing factor they are presumed to be idiopathic.

The patients received potassium citrate and potassium magnesium citrate supplementation after diagnosis of hypocitraturia. Even after 3 months of citrate supplementation (around 40-80 meq) along with dietary restrictions, it was difficult to correct the hypocitraturia. Only 7 of the 71 patients who had undergone a repeat urinary citrate level could achieve the normal desired range.

Hyperoxaluria was noted in 10 patients .4 of these patients also had concomitant hypocitraturia .In the absence of any renal failure, history of prior bowel rejection and other congenital diseases, Hyperoxaluria is believed to be secondary to increased oxalate consumptions.

Hypercalciuria was noted in 5 patients. However primary hyperparathyroidism was diagnosed in only one patient.

The most common stone was oxalate stone which comprised more than $83 \%$ in our study population. Uric acid stone is rare in our population and seen in only one patient.

Our study is limited by its retrospective nature. Secondly we do not have detailed information about the etiology of the hypocitraturia /hyperoxaluria. Detailed work up into the etiology is warranted.

Thus to summarize, in this study we noted that hypocitraturia is the most common predisposing factor for patients with incident renal stone. The hypocitraturia is mostly idiopathic in nature and does not improve even with standard treatment suggesting that the standard citrate supplementation may not be able to correct the biochemical abnormalities. Isolated hyperoxaluria and hypercalciuria are uncommon predisposing factors in our study population.

\section{Reference}

1. Pietro Manuel Ferraro, Eric N Taylor, Brian H Eisner, History of Kidney Stones and the Risk of Coronary Heart Disease, AMA 2013;310(4):408-415
2. Eric $\mathrm{N}$ taylor, Diane Feskanich, Julie $\mathrm{M}$ Paik, Gary C Curhan. Nephrolithiasis and risk of incident bone fracture, The Journal of urology 2016 may;195(5):1482-1486

3. Andrew D Rule, John C. Lieske, Xujian Li, L Joseph Melton: The ROKS Nomogram for Predicting a Second Symptomatic Stone Episode JASN 2014 Dec :25(12):2878-2886

4. Hamm LL, Renal handling of citrate. Kidney Int,1990,38:728-735

5. Simpson DP. Citrate excretion a window on renal metabolism. Am J physio; 1983,244:234-244

6. Jack M Zuckerman, BS, Dean G assimos, Hypocitraturia: Pathophysiology and Medical Management, reviews in Urology Vol 11 no.3 2009:134-144

7. Coe Fl, Parks JH Stone disease in hereditary distal renal tubular acidosis. Ann Intern Med. 1980; 93:60-61.

8. Hamm LL, Hering-Smith KS. Pathophysiology of hypocitraturic nephrolithiasis. Endocrinol Metab Clin North Am. 2002;31:885-893,

9. Rudman D, Dedonis JL, Fountain MT, et al. Hypocitraturia in patients with gastrointestinal malabsorption. N Engl J Med. 1980;303:657-661.

10. Pak CY. Citrate and renal calculi: an update. Miner Electrolyte Metab. 1994; 20:371-377.

11. Breslau NA, Brinkley L, Hill KD, Pak CY. Relationship of animal protein-rich diet to kidney stone formation and calcium metabolism. J Clin Endocrinol Metab. 1988;66:140-146.

12. Reddy ST, Wang CY, Sakhaee K, et al. Effect of low-carbohydrate high-protein diets on acidbase balance, stone-forming propensity, and calcium metabolism. Am J Kidney Dis. 2002; 40:265-274.

13. Gordon EE, Sheps SG. Effect of acetazolamide on citrate excretion and 
formation of renal calculi. N Engl J Med. 1957;256:1215-1219.

14. Melnick JZ, Preisig PA, Haynes S, et al. Converting enzyme inhibition causes hypocitraturia independent of acidosis or hypokalemia. Kidney Int. 1998;54:16701674.

15. Wright EM, Wright SH, Hirayama B, Kippen I. Interactions between lithium and renal transport of Krebs cycle intermediates. Proc Natl Acad Sci U S A. 1982; 79:7514-7517. 